CRYSTALLOGRAPHIC COMMUNICATIONS

ISSN 2056-9890

Received 22 June 2020

Accepted 6 July 2020

Edited by W. T. A. Harrison, University of Aberdeen, Scotland

Keywords: crystal structure; dihydrobenzoxazine; Hirshfeld surface.

CCDC reference: 2014264

Supporting information: this article has supporting information at journals.iucr.org/e

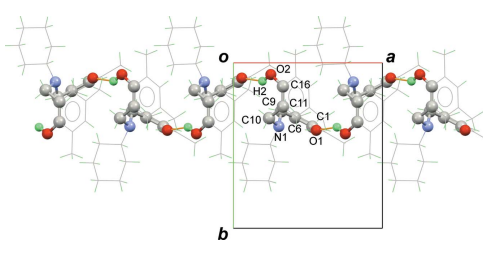

\section{Crystal structure and Hirshfeld surface analysis of the product of the ring-opening reaction of a dihydrobenzoxazine: 6,6'-[(cyclohexylazanediyl)- bis(methylene)]bis(2,4-dimethylphenol)}

\author{
Suttipong Wannapaiboon, ${ }^{a}$ Yuranan Hanlumyuang, ${ }^{\text {b }}$ Kantapat Chansaenpak, ${ }^{\text {c }}$ \\ Piyanut Pinyou, ${ }^{\mathrm{d}}$ Chatchai Veranitisagul, ${ }^{\mathrm{e}}$ Apirat Laobuthee ${ }^{\mathrm{b}}$ and Worawat \\ Wattanathana ${ }^{b_{*}}$ \\ aSynchrotron Light Research Institute, 111 University Avenue, Suranaree, Muang, Nakhon Ratchasima 30000, Thailand,
${ }^{\mathbf{b}}$ Department of Materials Engineering, Faculty of Engineering, Kasetsart University 10900, Thailand, 'National
Nanotechnology Center, National Science and Technology Development Agency, Thailand Science Park, Pathum Thani,
12120 , Thailand, ' $\mathbf{d}$ School of Chemistry, Institute of Science, Suranaree University of Technology, 111 University Avenue,
Suranaree, Muang, Nakhon Ratchasima 30000, Thailand, and ${ }^{\mathbf{e}}$ Department of Materials and Metallurgical Engineering,
Faculty of Engineering, Rajamangala University of Technology Thanyaburi, Pathumthani 12110, Thailand. *Correspon-
dence e-mail: fengwwwa@ku.ac.th
}

In the title unsymmetrical tertiary amine, $\mathrm{C}_{24} \mathrm{H}_{33} \mathrm{NO}_{2}$, which arose from the ringopening reaction of a dihydrobenzoxazine, two 2,4-dimethylphenol moieties are linked by a $6,6^{\prime}$-(cyclohexylazanediyl)-bis(methylene) bridge: the dihedral angle between the dimethylphenol rings is $72.45(7)^{\circ}$. The cyclohexyl ring adopts a chair conformation with the exocyclic $\mathrm{C}-\mathrm{N}$ bond in an equatorial orientation. One of the phenol $\mathrm{OH}$ groups forms an intramolecular $\mathrm{O}-\mathrm{H} \cdots \mathrm{N}$ hydrogen bond, generating an $S(6)$ ring, and a short intramolecular $\mathrm{C}-\mathrm{H} \cdots \mathrm{O}$ contact is also present. In the crystal, $\mathrm{O}-\mathrm{H} \cdots \mathrm{O}$ hydrogen bonds link the molecules into $C(10)$ chains propagating along the [100] direction. The Hirshfeld surface analysis of the title compound confirms the presence of these intra- and intermolecular interactions. The corresponding fingerprint plots indicate that the most significant contacts in the crystal packing are $\mathrm{H} \cdots \mathrm{H}(76.4 \%), \mathrm{H} \cdots \mathrm{C} /$ C.. $\mathrm{H}(16.3 \%)$, and $\mathrm{H} \cdots \mathrm{O} / \mathrm{O} \cdots \mathrm{H}(7.2 \%)$.

\section{Chemical context}

Dihydro-benzoxazines contain a benzene ring fused with a dihydro-oxazine ring (a six-membered heterocycle containing one nitrogen atom and one oxygen atom). Several isomers of dihydro-benzoxazines can be formed by varying the heteroatomic positions within the dihydro-oxazine ring. Among the different isomers of dihydro-benzoxazines, only 3,4-dihydro$2 H$-benzo[ $e$ ]-1,3-oxaxines (commonly called 1,3-2 $H$-benzoxazine monomers) can undergo a ring-opening polymerization reaction to form polybenzoxazines. As a result of various promising physical and chemical properties, polybenzoxazines have been studied by a number of workers (Ishida \& Allen, 1996; Ishida \& Agag 2011; Kiskan et al., 2011; Demir et al., 2013; Kim \& \& Ishida, 2001; Velez-Herrera et al., 2008; Xu et al., 2018). Moreover, a ring-opening polymerization to form the aza-methylene-phenol [-NR- $\left.\mathrm{CH}_{2}-\mathrm{C}_{6} \mathrm{H}_{4}(\mathrm{OH})-\right]$ moiety provides such hydrogen bonding as to interconnect with other materials (Froimowicz et al., 2016; Iguchi et al., 2018).

Interestingly, the use of phenol derivatives as initiators for the ring-opening polymerization of 3,4-dihydro- $2 \mathrm{H}$-benzo[ $e]$ 1,3-oxaxines leads to the formation of small molecules instead 
of polybenzoxazines (Chirachanchai et al., 2009). These small molecules (so-called dihydro-benzoxazine dimers), which generally possess an aza-methylene-phenol group, have been employed as models for describing polybenzoxazines (Hemvichian et al., 2002). In addition, the asymmetric Mannich reaction of the derivatives of dihydro-benzoxazine dimers, where only one $\mathrm{OH}$ group undergoes the ring-closure reaction has been reported (Laobuthee et al., 2001). As a result of these aza-methylene-phenol moieties, intermolecular and intramolecular hydrogen bonds are found in both the polybenzoxazines and the dihydro-benzoxazine dimers. They enhance the reactivity of the dihydro-benzoxazine dimers towards transition and rare-earth metal ions with respect to the common phenolic compounds. For instances, dihydrobenzoxazine dimers have been reported to be good chelating agents (Iguchi et al., 2018) for cerium ions (Veranitisagul et al., 2011) and copper ions (Phongtamrug et al., 2006).<smiles>Cc1cc(C)c(O)c(CN(Cc2cc(C)cc(C)c2O)C2CCCCC2)c1</smiles>

In this work, as part of our ongoing studies in this area (Wattanathana et al., 2016), we report the synthesis, crystal structure and Hirshfeld surface analysis of the title compound, (I).

\section{Structural commentary}

Fig. 1 shows the molecular structure of (I), which crystallizes in space group $P n a 2_{1}$. The tertiary-amine nitrogen atom (N1)

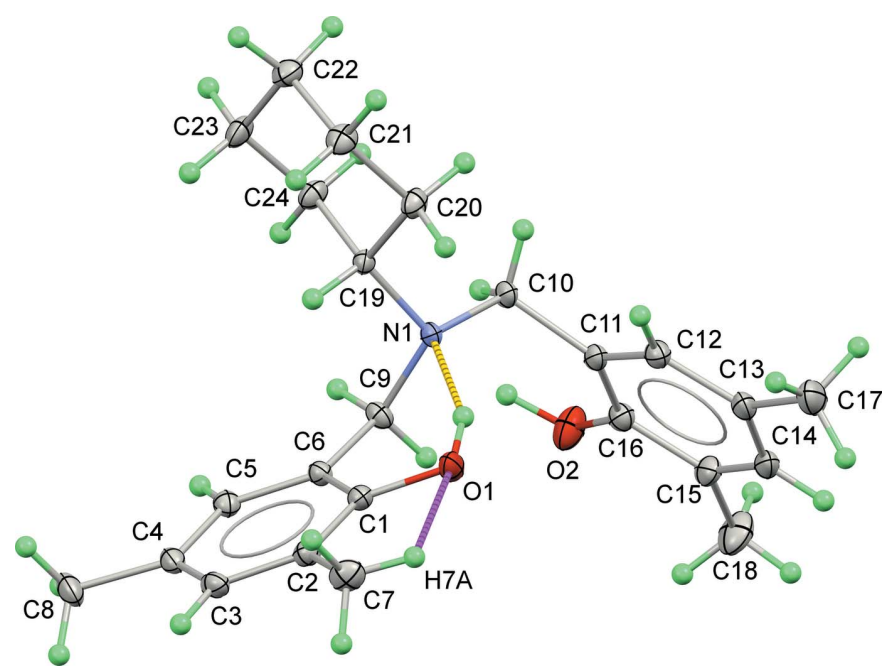

Figure 1

The molecular structure of (I) with displacement ellipsoids drawn at the $50 \%$ probability level. The $\mathrm{O}-\mathrm{H} \cdots \mathrm{O}$ and $\mathrm{C}-\mathrm{H} \cdots \mathrm{O}$ hydrogen bonds are shown as yellow and magenta dashed lines, respectively.
Table 1

Hydrogen-bond geometry $\left(\AA{ }^{\circ}\right)$.

\begin{tabular}{lllll}
\hline$D-\mathrm{H} \cdots A$ & $D-\mathrm{H}$ & $\mathrm{H} \cdots A$ & $D \cdots A$ & $D-\mathrm{H} \cdots A$ \\
\hline $\mathrm{O} 1-\mathrm{H} 1 \cdots \mathrm{N} 1$ & $0.89(4)$ & $1.81(4)$ & $2.630(2)$ & $153(3)$ \\
$\mathrm{O}^{\mathrm{i}}-\mathrm{H} 2 \cdots \mathrm{O} 1^{\mathrm{i}}$ & $0.99(4)$ & $1.87(4)$ & $2.741(2)$ & $145(3)$ \\
$\mathrm{C} 7-\mathrm{H} 7 A \cdots \mathrm{O} 1$ & 0.98 & 2.40 & $2.854(3)$ & 108
\end{tabular}

Symmetry code: (i) $x-\frac{1}{2},-y+\frac{1}{2}, z$.

adopts a distorted trigonal pyramidal shape because of the expansion of the angles around $\mathrm{N} 1$ atom $[\mathrm{C} 9-\mathrm{N} 1-\mathrm{C} 19=$ 112.59 (15); $\mathrm{C} 10-\mathrm{N} 1-\mathrm{C} 9=109.97$ (15); $\mathrm{C} 10-\mathrm{N} 1-\mathrm{C} 19=$ $115.09(15)$; bond-angle sum $=337.7^{\circ}$ ].

The non-hydrogen atoms of the 2,4-dimethylphenol moieties, namely $\mathrm{C} 1-\mathrm{C} 8 / \mathrm{O} 1$ and $\mathrm{C} 11-\mathrm{C} 18 / \mathrm{O} 2$, are almost planar (r.m.s. deviations $=0.030$ and $0.017 \AA$, respectively) and their mean planes subtend a dihedral angle of $72.45(7)^{\circ}$. The $\mathrm{C}$ atoms in the methyl groups in the para-positions with respect to the $\mathrm{OH}$ groups deviate the most from the calculated mean planes with deviations of 0.043 (2) for C8 and -0.033 (2) $\AA$ for C17. The cyclohexyl group adopts a regular chair conformation as seen from the $\mathrm{C}-\mathrm{C}-\mathrm{C}$ bond angles, which are in the range $109.14(17)^{\circ}$ to $111.59(17)^{\circ}$. The hydrogen atom bonded to $\mathrm{C} 19$ (H19) is in the axial position to allow the bulkier group (N1 tertiary-amine nitrogen atom) to be located at the equatorial position.

According to freely refined positions of the O-bound hydrogen atoms ( $\mathrm{H} 1$ and $\mathrm{H} 2), \mathrm{H} 1$ points toward $\mathrm{N} 1$ to set up an intramolecular $\mathrm{O}-\mathrm{H} \cdots \mathrm{N}$ hydrogen bond with an $S(6)$ graph-set motif (Table 1). This type of intramolecular $\mathrm{O}-$ $\mathrm{H} \cdots \mathrm{N}$ hydrogen bond is commonly noticed in the compounds having $-\mathrm{OH}$ and azamethylene groups attached to the benzene ring in the ortho positions (Suramitr et al., 2020), especially dihydro-benzoxazine dimer derivatives (Veranitisagul et al., 2012; Wattanathana et al., 2012, 2016). In addition to the classical hydrogen bond, one of the hydrogen atoms on the methyl side chain at the ortho position to the $\mathrm{O} 1$ atom exhibits a $\mathrm{C} 7-\mathrm{H} 7 A \cdots \mathrm{O} 1$ close contact (Table 1$)$ The characteristics of specific interactions for compound (I) are displayed as a non-covalent interaction plot (NCIPLOT) (Johnson et al., 2010; Contreras-García et al., 2011) in Fig. S1 of the supporting information.

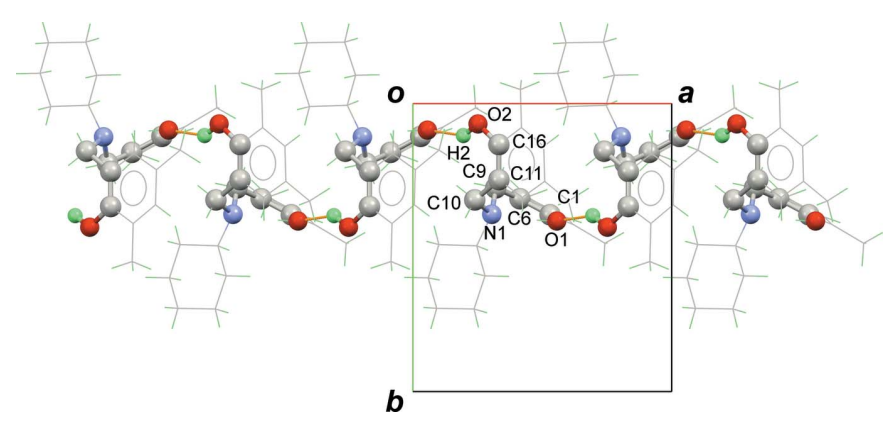

Figure 2

A view down [001] illustrating part of a [100] $C(10)$ chain of $\mathrm{O}-\mathrm{H} \cdots \mathrm{O}$ hydrogen bonds in the extended structure of (I). 


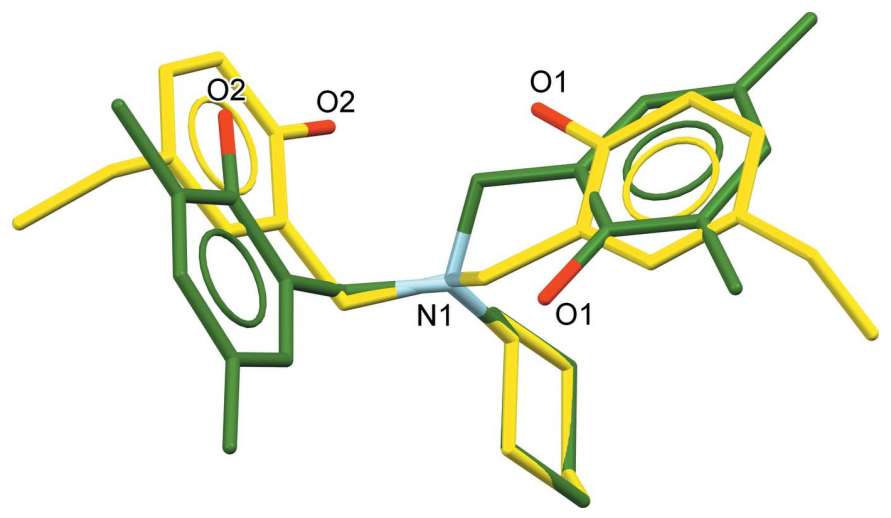

Figure 3

Overlay diagram of (I) (green structure) and its structural isomer (yellow structure, CEGYUK; Wattanathana et al., 2012). The N and six cyclohexyl $\mathrm{C}$ atoms are used as centers for structural overlay.

\section{Supramolecular features}

The other (O2) phenol group in (I) forms an intermolecular $\mathrm{O}-\mathrm{H} \cdots \mathrm{O}$ hydrogen bond with $\mathrm{O} 1$ as the acceptor, which generates $C(10)$ chains in the crystal, propagating in the [100] direction (Fig. 2). Unlike other dihydro-benzoxazine dimer derivatives, the title compound does not exhibit $R_{2}^{2}(20)$ hydrogen-bonded loops like those formed in 6,6'-(methylazanediyl)bis(methylene)bis(2,4-dimethylphenol) (NUPJOX: Dunkers et al., 1996; Phongtamrug et al., 2006; Veranitisagul et al., 2012a), 2,2'-(cyclohexylazanediyl)bis(methylene)bis(4ethylphenol) (SACYAZ and SADPEV; Wattanathana et al., 2016), 2,2'-(methylazanediyl)bis(methylene)bis(4-methylphenol) (IDUHEV; Wu et al., 2006), 2,2'-(methylazanediyl)bis(methylene)bis(4-methoxyphenol) (XEBBIR; Veranitisagul et al., 2012b), 2,2'-(cyclohexylazanediyl)bis(methylene)bis(4-methylphenol) (HETGOD; Phongtamrug et al., 2006), and 2,2'-(cyclohexylazanediyl)bis(methylene)bis(4ethylphenol) (CEGYUK; Wattanathana et al., 2012). This might be due to a greater steric effect from both the methyl and cyclohexyl groups.

The structure overlay of the title compound (green compound) and its structural isomer with only ethyl groups at the para-positions of the phenol rings (CEGYUK; Wattanathana et al., 2012) is displayed in Fig. 3. For CEGYUK, both $\mathrm{O} 1$ and $\mathrm{O} 2$ point toward the same side of the molecule to form the $R_{2}^{2}(20)$ hydrogen-bond motif just mentioned, while the O1 and $\mathrm{O} 2$ atoms of (I) are oriented in the opposite direction in order to reduce the steric effect. Therefore, the title molecules are joined together in an end-to-end packing mode into [100] chains (Fig. 2), where it may be seen that the bulky substituent groups are arrayed in an alternating fashion along the chain.

\section{Hirshfeld analysis}

To better understand and visualize the interactions within the crystal of the title compound, a Hirshfeld surface (HS) analysis (Spackman \& Jayatilaka, 2009) was carried out using Crystal Explorer 17.5 software (Turner et al., 2017). The HS plotted over the given range of $d_{\text {norm }}$ from -0.56 to 1.39 a.u.
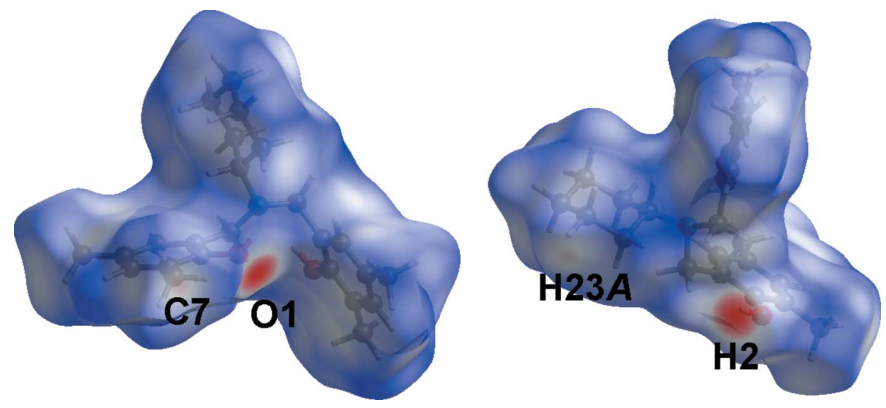

Figure 4

A view of the three-dimensional Hirshfeld surface of (I) plotted over $d_{\text {norm }}$ in the range -0.56 to 1.39 a.u.

(Fig. 4) shows faint red spots near $\mathrm{O} 1, \mathrm{H} 2$, and $\mathrm{C} 7$, confirming the $S(6)$ ring, $C(10)$ chain, and $\mathrm{C}-\mathrm{H} \cdots \mathrm{O}$ interaction, respectively.

Fig. 5 shows the full two-dimensional fingerprint plot and those delineated into individual interactions (McKinnon et al., 2007). The fingerprint plots show that the major contacts in the crystal structure are the contacts regarding $\mathrm{H}$ atoms only as the sum of all the H-related contributions is $99.9 \%$. The $\mathrm{H} \cdots \mathrm{H}$ contacts are characterized as a single spike at $d_{\mathrm{e}}+d_{\mathrm{i}} \simeq 2.3 \AA$ with the contribution of $76.4 \%$, while the $\mathrm{H} \cdots \mathrm{C} / \mathrm{C} \cdots \mathrm{H}$ contacts are observed as a pair of beak-shaped tips at $d_{\mathrm{e}}+d_{\mathrm{i}} \simeq$ $2.75 \AA$ with a contribution of $16.3 \%$. The pair of sharp peaks at $d_{\mathrm{e}}+d_{\mathrm{i}} \simeq 2.2 \AA$ represents the $\mathrm{H} \cdots \mathrm{O} / \mathrm{O} \cdots \mathrm{H}$ contacts $(7.2 \%)$. The C.C.C contact only participates slightly in the crystal packing as its individual contribution is only $0.1 \%$. The other contacts, i.e., $\mathrm{N} \cdots \mathrm{N}, \mathrm{H} \cdots \mathrm{N} / \mathrm{N} \cdots \mathrm{H}, \mathrm{C} \cdots \mathrm{N} / \mathrm{N} \cdots \mathrm{C}, \mathrm{C} \cdots \mathrm{O} /$

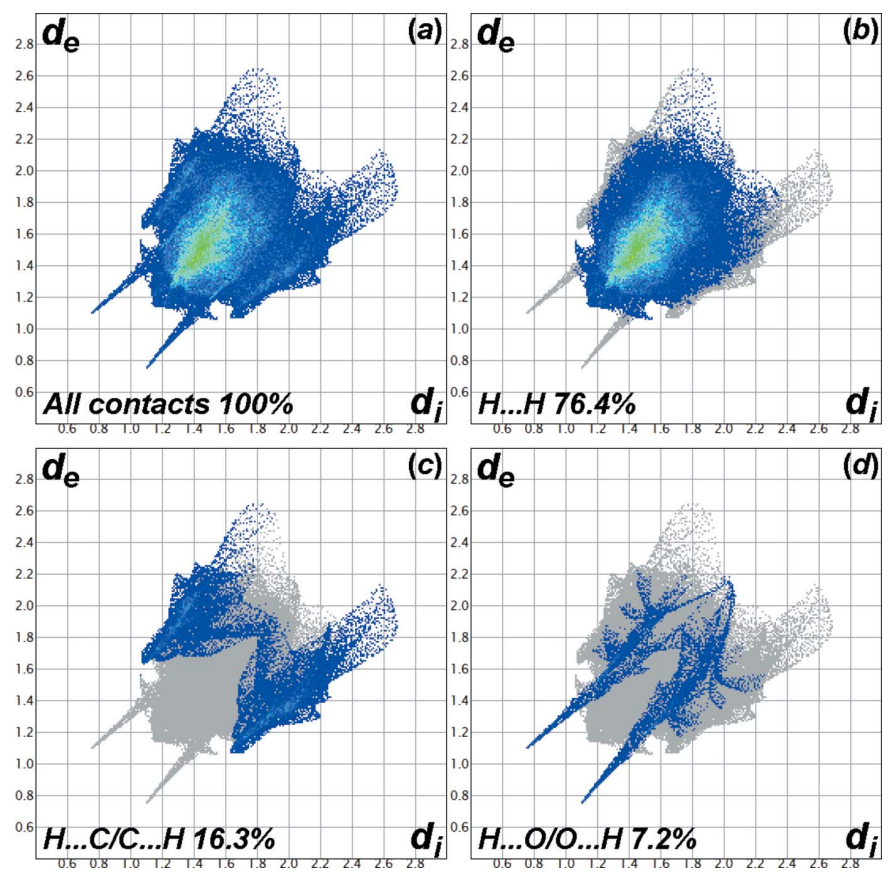

Figure 5

The full two-dimensional fingerprint plots for (I), showing (a) all interactions, and those delineated into $(b) \mathrm{H} \cdots \mathrm{H},(c) \mathrm{C} \cdots \mathrm{H} / \mathrm{H} \cdots \mathrm{C}$ and (d) $\mathrm{O} \cdots \mathrm{H} / \mathrm{H} \cdots \mathrm{O}$ interactions. 
$\mathrm{O} \cdots \mathrm{C}$, show no effect on the crystal packing due to the contribution of $0.0 \%$.

\section{Database survey}

A search for structures containing the bis(phenol) linked by a bis(methylene)aza bridge in the Cambridge Structural Database (CSD version 5.41, November 2019 + two updates; Groom et al., 2016) showed 156 match entries. Structural diversity of the dihydro-benzoxazine derivatives is observed as a result of the variation of the substituent groups on both the phenol moieties and tertiary-amine nitrogen atom. Several crystal structures of dihydro-benzoxazine dimer derivatives with no other substituent groups on both the phenol rings have been reported (BUZWUP; Abrahams et al., 2009; KEJRAU; Kuźnik et al., 2012). The crystal structures of dihydro-benzoxazine dimer derivatives with ortho substituents have also been reported, e.g., tert-butyl substituents (CIJLEN; Kelly et al., 2007) and methoxy substituents (SILROV; Liu et al., 2007). However, no crystal structures of dihydro-benzoxazine dimers possessing meta substituents have been reported. This might be due to the ortho and para directing property of the phenolic - $\mathrm{OH}$ groups. Dihydro-benzoxazine dimer derivatives with para substituents are very common, viz. with methyl groups (FANHOT; Janas et al., 2012, Singh et al., 2012; HETGOD; Phongtamrug et al., 2006; ICEMIO; Wang et al., 2011a, Rivera \& Bolte, 2016; IDUHEV; Wu et al., 2006; USODAC; Wang et al., 2011b,c), ethyl groups (CEGYUK; Wattanathana et al., 2012, SACYAZ and SADPEV; Wattanathana et al., 2016), a methoxy group (XEBBIR; Veranitisagul et al., 2012b), and tert-butyl groups (GIKJOC; Redjel et al. 2018). Apart from the monosubstituted derivatives, there are some reports on the crystal structures of ortho and para disubstituted derivatives, e.g., HEPZOU (Zhang et al., 2018) and RACMEP (Lionetti et al., 2010). Moreover, dihydrobenzoxazine dimers can also have different substituents on both the phenol rings as in AMEFUT, AMEGAA and AMEGEE (Sony et al., 2003), resulting in considerable structural variety.

When more restriction is applied to the search of 2,4-dimethylbis(phenol) linked by bis(methylene)aza bridge, the number of match structures is now reduced to 38 hits as only the $\mathrm{N}$-substituted grouping can change. Examples of different $\mathrm{N}$-substituents of the 6,6'-(azanediyl)bis(methylene)bis(2,4dimethylphenol) derivatives are $-\mathrm{CH}_{3}$ (NUPJOX; Dunkers et al., 1996, Phongtamrug et al., 2006, Veranitisagul et al., 2012a), $-\mathrm{CH}_{2} \mathrm{CH}_{2} \mathrm{OCH}_{3}$ (CAKDUP; Hasan et al., 2011), $-\mathrm{CH}_{2} \mathrm{CH}_{2} \mathrm{~N}\left(\mathrm{CH}_{3}\right)_{2}$ (ESAHUB; Velusamy et al., 2003, Lorber et al., 2005), $-\mathrm{CH}_{2} \mathrm{CH}_{2} \mathrm{CH}_{2} \mathrm{OH}$ (GIMWIL; Olesiejuk et al., 2018), $-\mathrm{CH}_{2} \mathrm{CH}_{2} \mathrm{CH}_{2} \mathrm{Cl}$ (GIMWOR; Olesiejuk et al., 2018), $-\mathrm{CH}_{2} \mathrm{CH}_{2} \mathrm{~N}\left(\mathrm{CH}_{2} \mathrm{CH}_{3}\right)_{2}$ (TOJSUI; Singh et al., 2012), and $-\mathrm{CH}_{2} \mathrm{CH}_{2} \mathrm{CH}_{2} \mathrm{~N}\left(\mathrm{CH}_{3}\right)_{2}$ (ZUXJAF; Bowser et al., 2016).

\section{Synthesis, characterization and crystallization}

Firstly, the corresponding dihydro-benzoxazine monomer, 3 -cyclohexyl-6,8-dimethyl-3,4-dihydro-2 $H$-benzo[ $e][1,3]$ oxa-
]$[1,3]$ oxazine, was prepared by a one-pot Mannich reaction (Chirachanchai et al., 2009; Wattanathana et al., 2014). Cyclohexylamine $(0.99 \mathrm{~g}, 10 \mathrm{mmol})$, paraformaldehyde $(0.63 \mathrm{~g}, 20 \mathrm{mmol})$ and 2,4-dimethylphenol $(1.22 \mathrm{~g}, 10 \mathrm{mmol})$ were dissolved in dioxane $(10 \mathrm{ml})$. The mixture was refluxed for $6 \mathrm{~h}$ to obtain a clear yellow solution. The solvent was removed by a rotary evaporator to obtain a yellowish viscous liquid as a crude product. After that, $10 \mathrm{ml}$ of dichloromethane were added to the dried crude product. The crude product was then washed by a liquid-liquid extraction method using $3 \mathrm{~N} \mathrm{NaOH}(10 \mathrm{ml})$ three times, followed by $10 \mathrm{ml}$ of deionized water for three more times until the solution became neutral. The product was dried by anhydrous sodium sulfate. Then, the dichloromethane solvent was removed by a rotary evaporator and consequently the dihydro-benzoxazine monomer, 3-cyclohexyl-6,8-dimethyl-3,4-dihydro- $2 \mathrm{H}$-benzo$[e][1,3]$ oxazine, (II), was collected.

An equimolar amount of 2,4-dimethylphenol was then mixed with (II) and the mixture was heated at $333 \mathrm{~K}$ overnight. After the reaction was complete, the yellow viscous liquid turned into a yellow solid, which was washed using diethyl ether, giving rise to a white precipitate of the title compound, which was separated from the yellow solution by decantation and rinsing with diethyl ether. The white precipitate was recrystallized from propan-2-ol solution to yield colourless blocks of (I).

M.p. $425 \mathrm{~K}$; FTIR (KBR pellet, $\left.\mathrm{cm}^{-1}\right)$ : $3384(b r, \mathrm{O}-\mathrm{H})$, $1484\left(v s, \mathrm{C}_{\mathrm{a}}-\mathrm{C}_{\mathrm{a}}\right), 1451\left(m, \mathrm{~N}-\mathrm{CH}_{3}\right), 1245(m, \mathrm{C}-\mathrm{N}), 1199(m$, $\mathrm{C}-\mathrm{N}-\mathrm{C}), 858(m, \mathrm{C}-\mathrm{N}-\mathrm{C})$; Raman $\left(\mathrm{cm}^{-1}\right): 3023\left(m, \mathrm{C}_{\mathrm{a}}-\right.$ $\mathrm{H}), 2942\left(v s, \mathrm{Csp}{ }^{3}-\mathrm{H}\right), 1447\left(m, \mathrm{~N}-\mathrm{CH}_{3}\right) ;{ }^{1} \mathrm{H}$ NMR $\left(\delta_{\mathrm{H}}\right.$, ppm): $1.06-1.14(m, 1 \mathrm{H}), 1.19(q, J=12.0 \mathrm{~Hz}, 2 \mathrm{H}), 1.44(q, J=$ $9.5 \mathrm{~Hz}, 2 \mathrm{H}), 1.64(d, J=12.0 \mathrm{~Hz}, 1 \mathrm{H}), 1.81(d, J=13.0 \mathrm{~Hz}, 2 \mathrm{H})$, $1.94(d, J=11.5 \mathrm{~Hz}, 2 \mathrm{H}), 2.21(d, J=11.0 \mathrm{~Hz}, 12 \mathrm{H}), 2.72(t t, J=$ $12.0,3.0 \mathrm{~Hz}, 1 \mathrm{H}), 3.73(s, 4 \mathrm{H}), 6.70(s, 2 \mathrm{H}), 6.85(s, 2 \mathrm{H}), 8.04(s$, $2 \mathrm{H}) ;{ }^{13} \mathrm{C}$ NMR $\left(\delta_{\mathrm{C}}, \mathrm{ppm}\right): 16.03\left(-\mathrm{CH}_{3}\right), 20.61\left(-\mathrm{CH}_{3}\right), 25.99$ $\left(\mathrm{C}_{\mathrm{cy}}\right), 26.35\left(\mathrm{C}_{\mathrm{cy}}\right), 27.66\left(\mathrm{C}_{\mathrm{cy}}\right), 51.64\left(-\mathrm{CH}_{2}-\mathrm{N} R_{2}\right), 57.65\left(\mathrm{C}_{\mathrm{cy}}-\right.$ $\left.\mathrm{N} R_{2}\right), 122.01\left(\mathrm{C}_{\mathrm{a}}\right), 124.94\left(\mathrm{C}_{\mathrm{a}}\right), 128.57\left(\mathrm{C}_{\mathrm{a}}\right), 128.65\left(\mathrm{C}_{\mathrm{a}}\right), 131.03$ $\left(\mathrm{C}_{\mathrm{a}}\right), 152.27(\mathrm{C}-\mathrm{OH})(\mathrm{cy}=$ cyclohexyl, $\mathrm{a}=$ aromatic $)$. Elemental analysis: analysis calculated for $\mathrm{C}_{24} \mathrm{H}_{33} \mathrm{NO}_{2}(\%)$ : C 78.47; H 8.99; N 3.82; found: C 78.49; H 8.97; N 3.85. The good agreement (see Fig. S2 in the supporting information) between the measured PXRD pattern of (I) and the calculated pattern based on the single crystal data indicates the high degree of crystal homogeneity and crystallinity of the obtained compound. For full details of the spectroscopic and powder diffraction measurements, see the supporting information.

\section{Refinement}

Crystal data, data collection and structure refinement details are summarized in Table 2. The O-bound $\mathrm{H}$ atoms ( $\mathrm{H} 1$ and $\mathrm{H} 2$ ) were located in a difference map and their positions were freely refined. The $\mathrm{C}$-bound $\mathrm{H}$ atoms were placed in idealized positions $(\mathrm{C}-\mathrm{H}=0.95-1.00 \AA$ depending on hybridization) and refined as riding atoms. The methyl groups were allowed to rotate, but not to tip, to best fit the electron density. The constraint $U_{\text {iso }}(\mathrm{H})=1.2 U_{\text {eq }}$ (carrier) or $1.5 U_{\text {eq }}$ (methyl C) was 
Table 2

Experimental details.

\begin{tabular}{|c|c|}
\hline \multicolumn{2}{|l|}{ Crystal data } \\
\hline Chemical formula & $\mathrm{C}_{24} \mathrm{H}_{33} \mathrm{NO}_{2}$ \\
\hline$M_{\mathrm{r}}$ & 367.51 \\
\hline Crystal system, space group & Orthorhombic, Pna $2_{1}$ \\
\hline Temperature $(\mathrm{K})$ & 100 \\
\hline$a, b, c(\AA)$ & $\begin{array}{l}10.2778(7), 11.4064(11) \\
17.5586(15)\end{array}$ \\
\hline$V\left(\AA^{3}\right)$ & $2058.4(3)$ \\
\hline$Z$ & 4 \\
\hline Radiation type & Мо $K \alpha$ \\
\hline$\mu\left(\mathrm{mm}^{-1}\right)$ & 0.07 \\
\hline Crystal size (mm) & $0.50 \times 0.28 \times 0.22$ \\
\hline \multicolumn{2}{|l|}{ Data collection } \\
\hline Diffractometer & Bruker APEXII CCD \\
\hline Absorption correction & $\begin{array}{l}\text { Multi-scan (SADABS; Bruker, } \\
\text { 2016) }\end{array}$ \\
\hline$T_{\min }, T_{\max }$ & $0.661,0.747$ \\
\hline $\begin{array}{l}\text { No. of measured, independent and } \\
\text { observed }[I>2 \sigma(I)] \text { reflections }\end{array}$ & $17289,7626,6367$ \\
\hline$R_{\text {int }}$ & 0.035 \\
\hline$(\sin \theta / \lambda)_{\max }\left(\AA^{-1}\right)$ & 0.771 \\
\hline \multicolumn{2}{|l|}{ Refinement } \\
\hline$R\left[F^{2}>2 \sigma\left(F^{2}\right)\right], w R\left(F^{2}\right), S$ & $0.053,0.139,1.04$ \\
\hline No. of reflections & 7626 \\
\hline No. of parameters & 256 \\
\hline No. of restraints & 1 \\
\hline H-atom treatment & $\begin{array}{l}\mathrm{H} \text { atoms treated by a mixture of } \\
\text { independent and constrained } \\
\text { refinement }\end{array}$ \\
\hline$\Delta \rho_{\max }, \Delta \rho_{\min }\left(\mathrm{e} \AA^{-3}\right)$ & $0.85,-0.26$ \\
\hline
\end{tabular}

Computer programs: APEX2 and SAINT (Bruker, 2018), SHELXT (Sheldrick, 2015a), SHELXL (Sheldrick, 2015b), OLEX2 (Dolomanov et al., 2009), Mercury (Macrae et al., 2020) and publCIF (Westrip, 2010).

applied in all cases. The absolute structure of (I) was indeterminate in the present refinement.

\section{Acknowledgements}

We thank the Department of Materials Engineering, Faculty of Engineering, Kasetsart University for the facility support. We acknowledge the Synchrotron Light Research Institute (Public Organization), SLRI, Thailand for the provision of beam time for XRD at BL1.1 W. All research staff of BL1.1 W are acknowledged for experimental consulting and assistance.

\section{References}

Abrahams, A., Gerber, T., Hosten, E. \& Mayer, P. (2009). Turk. J. Chem. 33, 569-577.

Bowser, A. K., Anderson-Wile, A. M., Johnston, D. H. \& Wile, B. M. (2016). Appl. Organomet. Chem. 30, 32-39.

Bruker (2016). SADABS. Bruker AXS Inc., Madison, Wisconsin, USA.

Bruker (2018). APEX2 and SAINT. Bruker AXS Inc., Madison, Wisconsin, USA.

Chirachanchai, S., Laobuthee, A. \& Phongtamrug, S. (2009). J. Heterocycl. Chem. 46, 714-721.

Contreras-García, J., Johnson, E. R., Keinan, S., Chaudret, R., Piquemal, J.-P., Beratan, D. N. \& Yang, W. (2011). J. Chem. Theory Comput. 7, 625-632.

Demir, K. D., Kiskan, B., Aydogan, B. \& Yagci, Y. (2013). React. Funct. Polym. 73, 346-359.
Dolomanov, O. V., Bourhis, L. J., Gildea, R. J., Howard, J. A. K. \& Puschmann, H. (2009). J. Appl. Cryst. 42, 339-341.

Dunkers, J., Zarate, E. A. \& Ishida, H. (1996). J. Phys. Chem. 100, 13514-13520.

Froimowicz, P., Zhang, K. \& Ishida, H. (2016). Chem. Eur. J. 22, 26912707.

Groom, C. R., Bruno, I. J., Lightfoot, M. P. \& Ward, S. C. (2016). Acta Cryst. B72, 171-179.

Hasan, K., Dawe, L. N. \& Kozak, C. N. (2011). Eur. J. Inorg. Chem. 2011, 4610-4621.

Hemvichian, K., Laobuthee, A., Chirachanchai, S. \& Ishida, H. (2002). Polym. Degrad. Stabil. 76, 1-15.

Iguchi, D., Salum, M. L. \& Froimowicz, P. (2018). Macromol. Chem. Phys. 220, 1800366.

Ishida, H. \& Agag, T. (2011). Handbook of Benzoxazine Resins. Amsterdam: Elsevier.

Ishida, H. \& Allen, D. J. (1996). J. Polym. Sci. B Polym. Phys. 34, 1019-1030.

Janas, Z., Nerkowski, T., Kober, E., Jerzykiewicz, L. B. \& Lis, T. (2012). Dalton Trans. 41, 442-447.

Johnson, E. R., Keinan, S., Mori-Sánchez, P., Contreras-García, J., Cohen, A. J. \& Yang, W. (2010). J. Am. Chem. Soc. 132, 6498-6506.

Kelly, B. V., Weintrob, E. C., Buccella, D., Tanski, J. M. \& Parkin, G. (2007). Inorg. Chem. Commun. 10, 699-704.

Kim, H. D. \& Ishida, H. (2001). J. Appl. Polym. Sci. 79, 1207-1219.

Kiskan, B., Ghosh, N. N. \& Yagci, Y. (2011). Polym. Int. 60, 167-177.

Kuźnik, N., Chrobaczyński, A., Mika, M., Miler, P., Komor, R. \& Kubicki, M. (2012). Eur. J. Med. Chem. 52, 184-192.

Laobuthee, A., Chirachanchai, S., Ishida, H. \& Tashiro, K. (2001). J. Am. Chem. Soc. 123, 9947-9955.

Lionetti, D., Medvecz, A. J., Ugrinova, V., Quiroz-Guzman, M., Noll, B. C. \& Brown, S. N. (2010). Inorg. Chem. 49, 4687-4697.

Liu, Y.-F., Xia, H.-T., Wang, D.-Q., Yang, S.-P. \& Meng, Y.-L. (2007). Acta Cryst. E63, o4070.

Lorber, C., Wolff, F., Choukroun, R. \& Vendier, L. (2005). Eur. J. Inorg. Chem. pp. 2850-2859.

Macrae, C. F., Sovago, I., Cottrell, S. J., Galek, P. T. A., McCabe, P., Pidcock, E., Platings, M., Shields, G. P., Stevens, J. S., Towler, M. \& Wood, P. A. (2020). J. Appl. Cryst. 53, 226-235.

McKinnon, J. J., Jayatilaka, D. \& Spackman, M. A. (2007). Chem. Commun. pp. 3814-3816.

Olesiejuk, M., Bakalorz, K., Krawczyk, T. \& Kuźnik, N. (2018). C. R. Chim. 21, 831-834.

Phongtamrug, S., Tashiro, K., Miyata, M. \& Chirachanchai, S. (2006). J. Phys. Chem. B, 110, 21365-21370.

Redjel, Y. K., Thevenin, L., Daran, J.-C., Benslimane, M., Poli, R. \& Fliedel, C. (2018). Polyhedron, 158, 83-90.

Rivera, A. \& Bolte, M. (2016). Private communication (refcode ICEMIO01). CCDC, Cambridge, England.

Sheldrick, G. M. (2015a). Acta Cryst. A71, 3-8.

Sheldrick, G. M. (2015b). Acta Cryst. C71, 3-8.

Singh, M., Butcher, R. J., Jasinski, J. P., Golen, J. A. \& Mugesh, G. (2012). J. Chem. Sci. 124, 1301-1313.

Sony, S. M. M., Kuppayee, M., Ponnuswamy, M. N., Manonmani, J., Kandaswamy, M. \& Fun, H.-K. (2003). J. Chem. Crystallogr. 33, 925-932.

Spackman, M. A. \& Jayatilaka, D. (2009). CrystEngComm, 11, 19-32.

Suramitr, S., Teanwarawat, J., Ithiapa, N., Wattanathana, W. \& Suramitr, A. (2020). Acta Cryst. E76, 1027-1032.

Turner, M. J., McKinnon, J. J., Wolff, S. K., Grimwood, D. J., Spackman, P. R., Jayatilaka, D. \& Spackman, M. A. (2017). Crystal Explorer 17. The University of Western Australia.

Velez-Herrera, P., Doyama, K., Abe, H. \& Ishida, H. (2008). Macromolecules, 41, 9704-9714.

Velusamy, M., Palaniandavar, M., Gopalan, R. S. \& Kulkarni, G. U. (2003). Inorg. Chem. 42, 8283-8293.

Veranitisagul, C., Kaewvilai, A., Duangthongyou, T., Koonsaeng, N. \& Laobuthee, A. (2012b). Acta Cryst. E68, o2139. 
Veranitisagul, C., Kaewvilai, A., Sangngern, S., Wattanathana, W., Suramitr, S., Koonsaeng, N. \& Laobuthee, A. (2011). Int. J. Mol. Sci. 12, 4365-4377.

Veranitisagul, C., Wattanathana, W., Kaewvilai, A., Duangthongyou, T., Laobuthee, A. \& Koonsaeng, N. (2012). Acta Cryst. E68, o1826.

Wang, N., Wang, Y., Li, J. \& Li, T. (2011c). Chin. J. Org. Chem. 31, 1703-1706.

Wang, N.-S., Wang, Y.-T., Guo, X.-K. \& Li, T.-D. (2011b). Acta Cryst. E67, o1438.

Wang, N.-S., Wang, Y.-T., Li, J.-D. \& Li, T.-D. (2011a). Chin. J. Struct. Chem. 30, 1533-1536.

Wattanathana, W., Nonthaglin, S., Veranitisagul, C., Koonsaeng, N. \& Laobuthee, A. (2014). J. Mol. Struct. 1074, 118-125.
Wattanathana, W., Nootsuwan, N., Veranitisagul, C., Koonsaeng, N., Suramitr, S. \& Laobuthee, A. (2016). J. Mol. Struct. 1109, 201208.

Wattanathana, W., Veranitisagul, C., Kaewvilai, A., Laobuthee, A. \& Koonsaeng, N. (2012). Acta Cryst. E68, o3050.

Westrip, S. P. (2010). J. Appl. Cryst. 43, 920-925.

Wu, M.-H., Liu, W.-J., Zou, W.-D. \& Wang, H.-Y. (2006). Acta Cryst. E62, o2949-02950.

Xu, Q., Zeng, M., Chen, J., Zeng, S., Huang, Y., Feng, Z., Xu, Q., Yan, C. \& Gu, Y. (2018). React. Funct. Polym. 122, 158-166.

Zhang, J., Wang, B., Wang, L., Sun, J., Zhang, Y., Cao, Z. \& Wu, Z. (2018). Appl. Organomet. Chem. 32, e4077. 


\section{supporting information}

Acta Cryst. (2020). E76, 1239-1244 [https://doi.org/10.1107/S2056989020009184]

Crystal structure and Hirshfeld surface analysis of the product of the ringopening reaction of a dihydrobenzoxazine: 6,6'-[(cyclohexylazanediyl)bis(methylene)]bis(2,4-dimethylphenol)

\section{Suttipong Wannapaiboon, Yuranan Hanlumyuang, Kantapat Chansaenpak, Piyanut Pinyou, Chatchai Veranitisagul, Apirat Laobuthee and Worawat Wattanathana}

Computing details

Data collection: APEX3 (Bruker, 2018); cell refinement: SAINT (Bruker, 2018); data reduction: SAINT(Bruker, 2018); program(s) used to solve structure: SHELXT (Sheldrick, 2015a); program(s) used to refine structure: SHELXL (Sheldrick, 2015b); molecular graphics: OLEX2 (Dolomanov et al., 2009), Mercury (Macrae et al., 2020); software used to prepare material for publication: publCIF (Westrip, 2010).

6,6'-[(Cyclohexylazanediyl)bis(methylene)]bis(2,4-dimethylphenol)

Crystal data

$\mathrm{C}_{24} \mathrm{H}_{33} \mathrm{NO}_{2}$

$M_{r}=367.51$

Orthorhombic, Pna2 1

$a=10.2778(7) \AA$

$b=11.4064$ (11) $\AA$

$c=17.5586(15) \AA$

$V=2058.4(3) \AA^{3}$

$Z=4$

$F(000)=800$

Data collection

Bruker APEXII CCD diffractometer

Graphite monochromator $\varphi$ and $\omega$ scans

Absorption correction: multi-scan

(SADABS; Bruker, 2016)

$T_{\min }=0.661, T_{\max }=0.747$

17289 measured reflections

Refinement

Refinement on $F^{2}$

Least-squares matrix: full

$R\left[F^{2}>2 \sigma\left(F^{2}\right)\right]=0.053$

$w R\left(F^{2}\right)=0.139$

$S=1.04$

7626 reflections

256 parameters
$D_{\mathrm{x}}=1.186 \mathrm{Mg} \mathrm{m}^{-3}$

Mo $K \alpha$ radiation, $\lambda=0.71073 \AA$

Cell parameters from 4297 reflections

$\theta=2.3-30.4^{\circ}$

$\mu=0.07 \mathrm{~mm}^{-1}$

$T=100 \mathrm{~K}$

Block, colourless

$0.50 \times 0.28 \times 0.22 \mathrm{~mm}$

7626 independent reflections 6367 reflections with $I>2 \sigma(I)$

$R_{\text {int }}=0.035$

$\theta_{\max }=33.2^{\circ}, \theta_{\min }=2.7^{\circ}$

$h=-15 \rightarrow 13$

$k=-17 \rightarrow 16$

$l=-26 \rightarrow 26$

\section{1 restraint}

Primary atom site location: dual

Hydrogen site location: mixed

$\mathrm{H}$ atoms treated by a mixture of independent and constrained refinement

$w=1 /\left[\sigma^{2}\left(F_{\mathrm{o}}^{2}\right)+(0.0816 P)^{2}+0.0042 P\right]$

where $P=\left(F_{\mathrm{o}}^{2}+2 F_{\mathrm{c}}^{2}\right) / 3$ 
$(\Delta / \sigma)_{\max }<0.001$

$\Delta \rho_{\max }=0.85$ e $\AA^{-3}$

$$
\Delta \rho_{\min }=-0.26 \text { e } \AA^{-3}
$$

Special details

Geometry. All esds (except the esd in the dihedral angle between two 1.s. planes) are estimated using the full covariance matrix. The cell esds are taken into account individually in the estimation of esds in distances, angles and torsion angles; correlations between esds in cell parameters are only used when they are defined by crystal symmetry. An approximate (isotropic) treatment of cell esds is used for estimating esds involving l.s. planes.

Fractional atomic coordinates and isotropic or equivalent isotropic displacement parameters $\left(\AA^{2}\right)$

\begin{tabular}{|c|c|c|c|c|}
\hline & $x$ & $y$ & $z$ & $U_{\text {iso }} * / U_{\text {eq }}$ \\
\hline $\mathrm{O} 1$ & $0.55606(15)$ & $0.40779(15)$ & $0.48635(9)$ & 0.0185 \\
\hline $\mathrm{O} 2$ & $0.25137(17)$ & $0.07066(16)$ & $0.38097(11)$ & $0.0282(4)$ \\
\hline N1 & $0.30494(16)$ & $0.38434(15)$ & $0.46177(9)$ & 0.0124 \\
\hline C11 & $0.33237(18)$ & $0.26439(18)$ & $0.34561(11)$ & 0.0146 \\
\hline $\mathrm{C} 1$ & 0.53009 (19) & $0.38603(17)$ & $0.56149(11)$ & $0.0136(3)$ \\
\hline $\mathrm{C} 4$ & $0.4756(2)$ & $0.34173(18)$ & $0.71588(11)$ & $0.0162(4)$ \\
\hline C16 & $0.33219(19)$ & 0.14251 (19) & $0.34158(12)$ & $0.0163(4)$ \\
\hline C6 & $0.41375(18)$ & $0.33056(18)$ & $0.58231(10)$ & $0.0129(3)$ \\
\hline $\mathrm{C} 5$ & $0.3869(2)$ & $0.31068(18)$ & $0.65887(11)$ & 0.0148 \\
\hline H5 & 0.306648 & 0.275282 & 0.672746 & $0.018 *$ \\
\hline C9 & 0.31994 (19) & $0.29297(18)$ & $0.52109(11)$ & $0.0148(4)$ \\
\hline H9A & 0.351767 & 0.219736 & 0.497244 & $0.018^{*}$ \\
\hline H9B & 0.234101 & 0.276476 & 0.544316 & $0.018 *$ \\
\hline C19 & $0.24654(17)$ & 0.49405 (17) & $0.49191(11)$ & $0.0118(3)$ \\
\hline H19 & 0.290794 & 0.509791 & 0.541586 & $0.014 *$ \\
\hline C10 & 0.23905 (18) & $0.33456(18)$ & $0.39420(11)$ & $0.0144(3)$ \\
\hline H10A & 0.201570 & 0.398953 & 0.363412 & $0.017^{*}$ \\
\hline H10B & 0.166781 & 0.283321 & 0.410990 & $0.017^{*}$ \\
\hline $\mathrm{C} 3$ & $0.5912(2)$ & 0.39448 (18) & $0.69330(12)$ & $0.0170(4)$ \\
\hline H3 & 0.653425 & 0.414046 & 0.731218 & $0.020 *$ \\
\hline $\mathrm{C} 24$ & 0.10054 (19) & 0.48969 (19) & $0.50922(12)$ & $0.0170(4)$ \\
\hline $\mathrm{H} 24 \mathrm{~A}$ & 0.081486 & 0.423062 & 0.543590 & $0.020 *$ \\
\hline H24B & 0.051024 & 0.478236 & 0.461434 & $0.020 *$ \\
\hline C14 & $0.5057(2)$ & $0.1484(2)$ & $0.24962(12)$ & $0.0201(4)$ \\
\hline H14 & 0.564743 & 0.108269 & 0.217144 & $0.024 *$ \\
\hline C13 & 0.50935 (19) & $0.2702(2)$ & $0.25194(12)$ & $0.0182(4)$ \\
\hline $\mathrm{C} 12$ & $0.4226(2)$ & $0.32580(19)$ & $0.30100(12)$ & $0.0167(4)$ \\
\hline H12 & 0.424682 & 0.408903 & 0.304371 & $0.020 *$ \\
\hline $\mathrm{C} 2$ & 0.61996 (19) & $0.42005(18)$ & $0.61731(12)$ & $0.0153(4)$ \\
\hline $\mathrm{C} 20$ & 0.27874 (19) & $0.59824(18)$ & $0.44034(12)$ & $0.0165(4)$ \\
\hline $\mathrm{H} 20 \mathrm{~A}$ & 0.373603 & 0.600307 & 0.430636 & $0.020 *$ \\
\hline $\mathrm{H} 20 \mathrm{~B}$ & 0.233910 & 0.588665 & 0.390821 & $0.020 *$ \\
\hline $\mathrm{C} 15$ & $0.4194(2)$ & 0.08345 (19) & $0.29282(13)$ & $0.0191(4)$ \\
\hline $\mathrm{C} 22$ & 0.09195 (19) & $0.7111(2)$ & $0.49721(13)$ & $0.0199(4)$ \\
\hline $\mathrm{H} 22 \mathrm{~A}$ & 0.039773 & 0.707735 & 0.449863 & $0.024 *$ \\
\hline $\mathrm{H} 22 \mathrm{~B}$ & 0.068791 & 0.784064 & 0.524558 & $0.024 *$ \\
\hline $\mathrm{C} 21$ & $0.2364(2)$ & $0.71323(18)$ & $0.47706(13)$ & $0.0187(4)$ \\
\hline
\end{tabular}




$\begin{array}{lllll}\text { H21A } & 0.253598 & 0.778781 & 0.441510 & 0.022^{*} \\ \text { H21B } & 0.288055 & 0.726786 & 0.523825 & 0.022^{*} \\ \text { C23 } & 0.0594(2) & 0.60523(19) & 0.54719(13) & 0.0197(4) \\ \text { H23A } & -0.035431 & 0.603583 & 0.557053 & 0.04^{*} \\ \text { H23B } & 0.104440 & 0.613096 & 0.596767 & 0.024^{*} \\ \text { C7 } & 0.7421(2) & 0.4850(2) & 0.59612(14) & 0.0216(4) \\ \text { H7A } & 0.752890 & 0.483810 & 0.540676 & 0.032^{*} \\ \text { H7B } & 0.817154 & 0.447173 & 0.620209 & 0.032^{*} \\ \text { H7C } & 0.735771 & 0.566363 & 0.613687 & 0.032^{*} \\ \text { C8 } & 0.4433(2) & 0.3241(2) & 0.79879(12) & 0.0228(4) \\ \text { H8A } & 0.412900 & 0.398284 & 0.820627 & 0.034^{*} \\ \text { H8B } & 0.521270 & 0.297887 & 0.826066 & 0.034^{*} \\ \text { H8C } & 0.374907 & 0.264769 & 0.803607 & 0.034^{*} \\ \text { C17 } & 0.6012(2) & 0.3382(3) & 0.20141(13) & 0.0265(5) \\ \text { H17A } & 0.681173 & 0.292866 & 0.193814 & 0.040^{*} \\ \text { H17B } & 0.622402 & 0.413246 & 0.225565 & 0.040^{*} \\ \text { H17C } & 0.559676 & 0.352574 & 0.152043 & 0.040^{*} \\ \text { C18 } & 0.4156(3) & -0.0484(2) & 0.28772(17) & 0.0318(5) \\ \text { H18A } & 0.418848 & -0.081899 & 0.339082 & 0.048^{*} \\ \text { H18B } & 0.490459 & -0.076175 & 0.258236 & 0.048^{*} \\ \text { H18C } & 0.334966 & -0.072901 & 0.262519 & 0.048^{*} \\ \text { H1 } & 0.480(3) & 0.405(3) & 0.463(2) & 0.042(10)^{*} \\ \text { H2 } & 0.195(4) & 0.111(4) & 0.419(3) & 0.057(12)^{*} \\ & & & & \end{array}$

Atomic displacement parameters $\left(\AA^{2}\right)$

\begin{tabular}{lllllll}
\hline & $U^{11}$ & $U^{22}$ & $U^{33}$ & $U^{12}$ & $U^{13}$ & $U^{23}$ \\
\hline $\mathrm{O} 1$ & $0.0135(6)$ & $0.0288(8)$ & $0.0133(6)$ & $0.0015(6)$ & $0.0020(5)$ & $0.0010(6)$ \\
$\mathrm{O} 2$ & $0.0312(9)$ & $0.0236(8)$ & $0.0299(9)$ & $-0.0038(7)$ & $0.0126(7)$ & $-0.0037(7)$ \\
$\mathrm{N} 1$ & $0.0136(7)$ & $0.0141(7)$ & $0.0094(6)$ & $0.0016(6)$ & $-0.0021(5)$ & $-0.0020(5)$ \\
$\mathrm{C} 11$ & $0.0150(8)$ & $0.0172(9)$ & $0.0116(7)$ & $0.0026(7)$ & $-0.0019(6)$ & $-0.0034(7)$ \\
$\mathrm{C} 1$ & $0.0133(8)$ & $0.0144(8)$ & $0.0132(8)$ & $0.0025(7)$ & $0.0000(6)$ & $-0.0009(7)$ \\
$\mathrm{C} 4$ & $0.0221(9)$ & $0.0144(9)$ & $0.0120(8)$ & $0.0034(7)$ & $-0.0018(7)$ & $-0.0004(7)$ \\
$\mathrm{C} 16$ & $0.0165(8)$ & $0.0175(9)$ & $0.0148(8)$ & $0.0002(7)$ & $-0.0016(6)$ & $-0.0024(7)$ \\
$\mathrm{C} 6$ & $0.0135(8)$ & $0.0136(8)$ & $0.0116(8)$ & $0.0013(6)$ & $-0.0021(6)$ & $-0.0007(6)$ \\
$\mathrm{C} 5$ & $0.0176(8)$ & $0.0130(8)$ & $0.0138(8)$ & $0.0008(7)$ & $0.0000(6)$ & $0.0004(7)$ \\
$\mathrm{C} 9$ & $0.0165(8)$ & $0.0153(9)$ & $0.0125(8)$ & $-0.0014(7)$ & $-0.0028(6)$ & $0.0001(7)$ \\
$\mathrm{C} 19$ & $0.0122(7)$ & $0.0128(8)$ & $0.0104(7)$ & $0.0005(6)$ & $0.0012(6)$ & $-0.0010(6)$ \\
$\mathrm{C} 10$ & $0.0149(8)$ & $0.0169(9)$ & $0.0115(7)$ & $0.0019(7)$ & $-0.0029(6)$ & $-0.0044(7)$ \\
$\mathrm{C} 3$ & $0.0175(8)$ & $0.0178(9)$ & $0.0158(8)$ & $0.0036(7)$ & $-0.0057(7)$ & $-0.0038(7)$ \\
$\mathrm{C} 24$ & $0.0143(8)$ & $0.0166(9)$ & $0.0200(9)$ & $0.0006(7)$ & $0.0045(7)$ & $-0.0001(7)$ \\
C14 & $0.0196(9)$ & $0.0279(11)$ & $0.0127(8)$ & $0.0080(8)$ & $-0.0023(7)$ & $-0.0046(8)$ \\
C13 & $0.0174(8)$ & $0.0263(10)$ & $0.0110(8)$ & $0.0036(8)$ & $-0.0007(7)$ & $-0.0007(8)$ \\
C12 & $0.0193(9)$ & $0.0171(9)$ & $0.0135(8)$ & $0.0021(7)$ & $-0.0019(7)$ & $-0.0013(7)$ \\
C2 & $0.0132(8)$ & $0.0156(9)$ & $0.0170(8)$ & $0.0013(7)$ & $-0.0016(6)$ & $-0.0026(7)$ \\
C20 & $0.0170(8)$ & $0.0158(9)$ & $0.0166(8)$ & $0.0002(7)$ & $0.0048(7)$ & $0.0016(7)$ \\
C15 & $0.0230(9)$ & $0.0175(9)$ & $0.0167(9)$ & $0.0047(8)$ & $-0.0019(7)$ & $-0.0057(8)$ \\
C22 & $0.0193(9)$ & $0.0178(9)$ & $0.0224(10)$ & $0.0047(7)$ & $0.0018(7)$ & $-0.0010(7)$
\end{tabular}




\begin{tabular}{lllllll} 
C21 & $0.0203(9)$ & $0.0146(9)$ & $0.0210(9)$ & $-0.0008(7)$ & $0.0026(7)$ & $0.0003(7)$ \\
C23 & $0.0179(9)$ & $0.0205(10)$ & $0.0209(10)$ & $0.0034(7)$ & $0.0079(7)$ & $-0.0023(8)$ \\
C7 & $0.0156(9)$ & $0.0235(10)$ & $0.0256(10)$ & $-0.0021(8)$ & $-0.0027(8)$ & $-0.0032(8)$ \\
C8 & $0.0334(11)$ & $0.0237(10)$ & $0.0113(8)$ & $0.0018(9)$ & $-0.0031(8)$ & $0.0007(8)$ \\
C17 & $0.0234(10)$ & $0.0382(13)$ & $0.0178(10)$ & $0.0042(10)$ & $0.0039(8)$ & $0.0044(9)$ \\
C18 & $0.0414(14)$ & $0.0193(11)$ & $0.0348(13)$ & $0.0026(10)$ & $0.0087(11)$ & $-0.0083(10)$ \\
\hline
\end{tabular}

Geometric parameters $\left(\AA,{ }^{o}\right)$

\begin{tabular}{|c|c|c|c|}
\hline $\mathrm{O} 1-\mathrm{C} 1$ & $1.369(2)$ & $\mathrm{C} 14-\mathrm{H} 14$ & 0.9500 \\
\hline $\mathrm{O} 1-\mathrm{H} 1$ & $0.89(4)$ & $\mathrm{C} 14-\mathrm{C} 13$ & $1.391(3)$ \\
\hline $\mathrm{O} 2-\mathrm{C} 16$ & $1.356(3)$ & $\mathrm{C} 14-\mathrm{C} 15$ & $1.382(3)$ \\
\hline $\mathrm{O} 2-\mathrm{H} 2$ & $0.99(4)$ & $\mathrm{C} 13-\mathrm{C} 12$ & $1.393(3)$ \\
\hline $\mathrm{N} 1-\mathrm{C} 9$ & $1.482(3)$ & $\mathrm{C} 13-\mathrm{C} 17$ & $1.510(3)$ \\
\hline $\mathrm{N} 1-\mathrm{C} 19$ & $1.485(2)$ & $\mathrm{C} 12-\mathrm{H} 12$ & 0.9500 \\
\hline $\mathrm{N} 1-\mathrm{C} 10$ & $1.479(2)$ & $\mathrm{C} 2-\mathrm{C} 7$ & $1.504(3)$ \\
\hline $\mathrm{C} 11-\mathrm{C} 16$ & $1.392(3)$ & $\mathrm{C} 20-\mathrm{H} 20 \mathrm{~A}$ & 0.9900 \\
\hline $\mathrm{C} 11-\mathrm{C} 10$ & $1.513(3)$ & $\mathrm{C} 20-\mathrm{H} 20 \mathrm{~B}$ & 0.9900 \\
\hline $\mathrm{C} 11-\mathrm{C} 12$ & $1.402(3)$ & $\mathrm{C} 20-\mathrm{C} 21$ & $1.525(3)$ \\
\hline $\mathrm{C} 1-\mathrm{C} 6$ & $1.401(3)$ & $\mathrm{C} 15-\mathrm{C} 18$ & $1.507(3)$ \\
\hline $\mathrm{C} 1-\mathrm{C} 2$ & $1.402(3)$ & $\mathrm{C} 22-\mathrm{H} 22 \mathrm{~A}$ & 0.9900 \\
\hline $\mathrm{C} 4-\mathrm{C} 5$ & $1.399(3)$ & $\mathrm{C} 22-\mathrm{H} 22 \mathrm{~B}$ & 0.9900 \\
\hline $\mathrm{C} 4-\mathrm{C} 3$ & $1.390(3)$ & $\mathrm{C} 22-\mathrm{C} 21$ & $1.526(3)$ \\
\hline $\mathrm{C} 4-\mathrm{C} 8$ & $1.506(3)$ & $\mathrm{C} 22-\mathrm{C} 23$ & $1.530(3)$ \\
\hline $\mathrm{C} 16-\mathrm{C} 15$ & $1.411(3)$ & $\mathrm{C} 21-\mathrm{H} 21 \mathrm{~A}$ & 0.9900 \\
\hline $\mathrm{C} 6-\mathrm{C} 5$ & $1.391(3)$ & $\mathrm{C} 21-\mathrm{H} 21 \mathrm{~B}$ & 0.9900 \\
\hline $\mathrm{C} 6-\mathrm{C} 9$ & $1.506(3)$ & $\mathrm{C} 23-\mathrm{H} 23 \mathrm{~A}$ & 0.9900 \\
\hline $\mathrm{C} 5-\mathrm{H} 5$ & 0.9500 & $\mathrm{C} 23-\mathrm{H} 23 \mathrm{~B}$ & 0.9900 \\
\hline C9-H9A & 0.9900 & $\mathrm{C} 7-\mathrm{H} 7 \mathrm{~A}$ & 0.9800 \\
\hline C9- & 0.9900 & $\mathrm{C} 7-\mathrm{H} 7 \mathrm{~B}$ & 0.9800 \\
\hline C19-H19 & 1.0000 & $\mathrm{C} 7-\mathrm{H} 7 \mathrm{C}$ & 0.9800 \\
\hline $\mathrm{C} 19-\mathrm{C} 24$ & $1.532(3)$ & $\mathrm{C} 8-\mathrm{H} 8 \mathrm{~A}$ & 0.9800 \\
\hline $\mathrm{C} 19-\mathrm{C} 20$ & $1.530(3)$ & $\mathrm{C} 8-\mathrm{H} 8 \mathrm{~B}$ & 0.9800 \\
\hline $\mathrm{C} 10-\mathrm{H} 10 \mathrm{~A}$ & 0.9900 & $\mathrm{C} 8-\mathrm{H} 8 \mathrm{C}$ & 0.9800 \\
\hline $\mathrm{C} 10-\mathrm{H} 10 \mathrm{~B}$ & 0.9900 & C17-H17A & 0.9800 \\
\hline $\mathrm{C} 3-\mathrm{H} 3$ & 0.9500 & C17-H17B & 0.9800 \\
\hline $\mathrm{C} 3-\mathrm{C} 2$ & $1.397(3)$ & $\mathrm{C} 17-\mathrm{H} 17 \mathrm{C}$ & 0.9800 \\
\hline $\mathrm{C} 24-\mathrm{H} 24 \mathrm{~A}$ & 0.9900 & $\mathrm{C} 18-\mathrm{H} 18 \mathrm{~A}$ & 0.9800 \\
\hline $\mathrm{C} 24-\mathrm{H} 24 \mathrm{~B}$ & 0.9900 & C18-H18B & 0.9800 \\
\hline $\mathrm{C} 24-\mathrm{C} 23$ & $1.536(3)$ & $\mathrm{C} 18-\mathrm{H} 18 \mathrm{C}$ & 0.9800 \\
\hline $\mathrm{C} 1-\mathrm{O} 1-\mathrm{H} 1$ & $106(2)$ & $\mathrm{C} 13-\mathrm{C} 12-\mathrm{C} 11$ & $122.80(19)$ \\
\hline $\mathrm{C} 16-\mathrm{O} 2-\mathrm{H} 2$ & $115(2)$ & $\mathrm{C} 13-\mathrm{C} 12-\mathrm{H} 12$ & 118.6 \\
\hline $\mathrm{C} 9-\mathrm{N} 1-\mathrm{C} 19$ & $112.59(15)$ & $\mathrm{C} 1-\mathrm{C} 2-\mathrm{C} 7$ & $120.89(19)$ \\
\hline $\mathrm{C} 10-\mathrm{N} 1-\mathrm{C} 9$ & $109.97(15)$ & $\mathrm{C} 3-\mathrm{C} 2-\mathrm{C} 1$ & $118.06(18)$ \\
\hline $\mathrm{C} 10-\mathrm{N} 1-\mathrm{C} 19$ & $115.09(15)$ & $\mathrm{C} 3-\mathrm{C} 2-\mathrm{C} 7$ & $121.03(18)$ \\
\hline $\mathrm{C} 16-\mathrm{C} 11-\mathrm{C} 10$ & $123.80(18)$ & $\mathrm{C} 19-\mathrm{C} 20-\mathrm{H} 20 \mathrm{~A}$ & 109.5 \\
\hline $\mathrm{C} 16-\mathrm{C} 11-\mathrm{C} 12$ & $118.14(18)$ & $\mathrm{C} 19-\mathrm{C} 20-\mathrm{H} 20 \mathrm{~B}$ & 109.5 \\
\hline
\end{tabular}




\begin{tabular}{|c|c|}
\hline $\mathrm{C} 12-\mathrm{C} 11-\mathrm{C} 10$ & $118.05(18)$ \\
\hline $\mathrm{O} 1-\mathrm{C} 1-\mathrm{C} 6$ & $119.99(17)$ \\
\hline $\mathrm{O} 1-\mathrm{C} 1-\mathrm{C} 2$ & $119.69(18)$ \\
\hline $\mathrm{C} 6-\mathrm{C} 1-\mathrm{C} 2$ & $120.32(18)$ \\
\hline $\mathrm{C} 5-\mathrm{C} 4-\mathrm{C} 8$ & $120.95(19)$ \\
\hline $\mathrm{C} 3-\mathrm{C} 4-\mathrm{C} 5$ & $117.54(19)$ \\
\hline $\mathrm{C} 3-\mathrm{C} 4-\mathrm{C} 8$ & $121.43(19)$ \\
\hline $\mathrm{O} 2-\mathrm{C} 16-\mathrm{C} 11$ & $125.33(19)$ \\
\hline $\mathrm{O} 2-\mathrm{C} 16-\mathrm{C} 15$ & $114.21(19)$ \\
\hline $\mathrm{C} 11-\mathrm{C} 16-\mathrm{C} 15$ & $120.46(19)$ \\
\hline $\mathrm{C} 1-\mathrm{C} 6-\mathrm{C} 9$ & $119.24(17)$ \\
\hline $\mathrm{C} 5-\mathrm{C} 6-\mathrm{C} 1$ & $119.68(18)$ \\
\hline $\mathrm{C} 5-\mathrm{C} 6-\mathrm{C} 9$ & $121.08(18)$ \\
\hline $\mathrm{C} 4-\mathrm{C} 5-\mathrm{H} 5$ & 119.3 \\
\hline $\mathrm{C} 6-\mathrm{C} 5-\mathrm{C} 4$ & $121.38(19)$ \\
\hline $\mathrm{C} 6-\mathrm{C} 5-\mathrm{H} 5$ & 119.3 \\
\hline $\mathrm{N} 1-\mathrm{C} 9-\mathrm{C} 6$ & $111.60(16)$ \\
\hline $\mathrm{N} 1-\mathrm{C} 9-\mathrm{H} 9 \mathrm{~A}$ & 109.3 \\
\hline $\mathrm{N} 1-\mathrm{C} 9-\mathrm{H} 9 \mathrm{~B}$ & 109.3 \\
\hline $\mathrm{C} 6-\mathrm{C} 9-\mathrm{H} 9 \mathrm{~A}$ & 109.3 \\
\hline $\mathrm{C} 6-\mathrm{C} 9-\mathrm{H} 9 \mathrm{~B}$ & 109.3 \\
\hline $\mathrm{H} 9 \mathrm{~A}-\mathrm{C} 9-\mathrm{H} 9 \mathrm{~B}$ & 108.0 \\
\hline N1-C19-H19 & 106.2 \\
\hline $\mathrm{N} 1-\mathrm{C} 19-\mathrm{C} 24$ & $116.06(16)$ \\
\hline $\mathrm{N} 1-\mathrm{C} 19-\mathrm{C} 20$ & $110.85(15)$ \\
\hline C24-C19-H19 & 106.2 \\
\hline $\mathrm{C} 20-\mathrm{C} 19-\mathrm{H} 19$ & 106.2 \\
\hline $\mathrm{C} 20-\mathrm{C} 19-\mathrm{C} 24$ & $110.76(16)$ \\
\hline $\mathrm{N} 1-\mathrm{C} 10-\mathrm{C} 11$ & $111.41(15)$ \\
\hline $\mathrm{N} 1-\mathrm{C} 10-\mathrm{H} 10 \mathrm{~A}$ & 109.3 \\
\hline $\mathrm{N} 1-\mathrm{C} 10-\mathrm{H} 10 \mathrm{~B}$ & 109.3 \\
\hline $\mathrm{C} 11-\mathrm{C} 10-\mathrm{H} 10 \mathrm{~A}$ & 109.3 \\
\hline $\mathrm{C} 11-\mathrm{C} 10-\mathrm{H} 10 \mathrm{~B}$ & 109.3 \\
\hline $\mathrm{H} 10 \mathrm{~A}-\mathrm{C} 10-\mathrm{H} 10 \mathrm{~B}$ & 108.0 \\
\hline $\mathrm{C} 4-\mathrm{C} 3-\mathrm{H} 3$ & 118.5 \\
\hline $\mathrm{C} 4-\mathrm{C} 3-\mathrm{C} 2$ & $122.94(18)$ \\
\hline $\mathrm{C} 2-\mathrm{C} 3-\mathrm{H} 3$ & 118.5 \\
\hline $\mathrm{C} 19-\mathrm{C} 24-\mathrm{H} 24 \mathrm{~A}$ & 109.9 \\
\hline $\mathrm{C} 19-\mathrm{C} 24-\mathrm{H} 24 \mathrm{~B}$ & 109.9 \\
\hline $\mathrm{C} 19-\mathrm{C} 24-\mathrm{C} 23$ & $109.14(17)$ \\
\hline $\mathrm{H} 24 \mathrm{~A}-\mathrm{C} 24-\mathrm{H} 24 \mathrm{~B}$ & 108.3 \\
\hline $\mathrm{C} 23-\mathrm{C} 24-\mathrm{H} 24 \mathrm{~A}$ & 109.9 \\
\hline $\mathrm{C} 23-\mathrm{C} 24-\mathrm{H} 24 \mathrm{~B}$ & 109.9 \\
\hline $\mathrm{C} 13-\mathrm{C} 14-\mathrm{H} 14$ & 118.8 \\
\hline $\mathrm{C} 15-\mathrm{C} 14-\mathrm{H} 14$ & 118.8 \\
\hline $\mathrm{C} 15-\mathrm{C} 14-\mathrm{C} 13$ & $122.5(2)$ \\
\hline $\mathrm{C} 14-\mathrm{C} 13-\mathrm{C} 12$ & $117.1(2)$ \\
\hline $\mathrm{C} 14-\mathrm{C} 13-\mathrm{C} 17$ & $120.9(2)$ \\
\hline
\end{tabular}

$\mathrm{H} 20 \mathrm{~A}-\mathrm{C} 20-\mathrm{H} 20 \mathrm{~B} \quad 108.1$

$\mathrm{C} 21-\mathrm{C} 20-\mathrm{C} 19 \quad 110.85$ (17)

$\mathrm{C} 21-\mathrm{C} 20-\mathrm{H} 20 \mathrm{~A} \quad 109.5$

$\mathrm{C} 21-\mathrm{C} 20-\mathrm{H} 20 \mathrm{~B} \quad 109.5$

$\mathrm{C} 16-\mathrm{C} 15-\mathrm{C} 18 \quad 119.7$ (2)

$\mathrm{C} 14-\mathrm{C} 15-\mathrm{C} 16 \quad 119.01$ (19)

$\mathrm{C} 14-\mathrm{C} 15-\mathrm{C} 18 \quad 121.2(2)$

$\mathrm{H} 22 \mathrm{~A}-\mathrm{C} 22-\mathrm{H} 22 \mathrm{~B} \quad 108.0$

$\mathrm{C} 21-\mathrm{C} 22-\mathrm{H} 22 \mathrm{~A} \quad 109.4$

$\mathrm{C} 21-\mathrm{C} 22-\mathrm{H} 22 \mathrm{~B} \quad 109.4$

$\mathrm{C} 21-\mathrm{C} 22-\mathrm{C} 23 \quad 111.00$ (18)

$\mathrm{C} 23-\mathrm{C} 22-\mathrm{H} 22 \mathrm{~A} \quad 109.4$

$\mathrm{C} 23-\mathrm{C} 22-\mathrm{H} 22 \mathrm{~B} \quad 109.4$

$\mathrm{C} 20-\mathrm{C} 21-\mathrm{C} 22 \quad 111.22$ (17)

$\mathrm{C} 20-\mathrm{C} 21-\mathrm{H} 21 \mathrm{~A} \quad 109.4$

$\mathrm{C} 20-\mathrm{C} 21-\mathrm{H} 21 \mathrm{~B} \quad 109.4$

$\mathrm{C} 22-\mathrm{C} 21-\mathrm{H} 21 \mathrm{~A} \quad 109.4$

$\mathrm{C} 22-\mathrm{C} 21-\mathrm{H} 21 \mathrm{~B} \quad 109.4$

$\mathrm{H} 21 \mathrm{~A}-\mathrm{C} 21-\mathrm{H} 21 \mathrm{~B} \quad 108.0$

$\mathrm{C} 24-\mathrm{C} 23-\mathrm{H} 23 \mathrm{~A} \quad 109.3$

$\mathrm{C} 24-\mathrm{C} 23-\mathrm{H} 23 \mathrm{~B} \quad 109.3$

$\mathrm{C} 22-\mathrm{C} 23-\mathrm{C} 24 \quad 111.59$ (17)

$\mathrm{C} 22-\mathrm{C} 23-\mathrm{H} 23 \mathrm{~A} \quad 109.3$

$\mathrm{C} 22-\mathrm{C} 23-\mathrm{H} 23 \mathrm{~B} \quad 109.3$

$\mathrm{H} 23 \mathrm{~A}-\mathrm{C} 23-\mathrm{H} 23 \mathrm{~B} \quad 108.0$

$\mathrm{C} 2-\mathrm{C} 7-\mathrm{H} 7 \mathrm{~A} \quad 109.5$

$\mathrm{C} 2-\mathrm{C} 7-\mathrm{H} 7 \mathrm{~B} \quad 109.5$

$\mathrm{C} 2-\mathrm{C} 7-\mathrm{H} 7 \mathrm{C} \quad 109.5$

$\mathrm{H} 7 \mathrm{~A}-\mathrm{C} 7-\mathrm{H} 7 \mathrm{~B} \quad 109.5$

$\mathrm{H} 7 \mathrm{~A}-\mathrm{C} 7-\mathrm{H} 7 \mathrm{C} \quad 109.5$

$\mathrm{H} 7 \mathrm{~B}-\mathrm{C} 7-\mathrm{H} 7 \mathrm{C} \quad 109.5$

$\mathrm{C} 4-\mathrm{C} 8-\mathrm{H} 8 \mathrm{~A} \quad 109.5$

$\mathrm{C} 4-\mathrm{C} 8-\mathrm{H} 8 \mathrm{~B} \quad 109.5$

$\mathrm{C} 4-\mathrm{C} 8-\mathrm{H} 8 \mathrm{C} \quad 109.5$

$\mathrm{H} 8 \mathrm{~A}-\mathrm{C} 8-\mathrm{H} 8 \mathrm{~B} \quad 109.5$

$\mathrm{H} 8 \mathrm{~A}-\mathrm{C} 8-\mathrm{H} 8 \mathrm{C} \quad 109.5$

$\mathrm{H} 8 \mathrm{~B}-\mathrm{C} 8-\mathrm{H} 8 \mathrm{C} \quad 109.5$

$\mathrm{C} 13-\mathrm{C} 17-\mathrm{H} 17 \mathrm{~A} \quad 109.5$

$\mathrm{C} 13-\mathrm{C} 17-\mathrm{H} 17 \mathrm{~B} \quad 109.5$

$\mathrm{C} 13-\mathrm{C} 17-\mathrm{H} 17 \mathrm{C} \quad 109.5$

H17A-C17-H17B $\quad 109.5$

$\mathrm{H} 17 \mathrm{~A}-\mathrm{C} 17-\mathrm{H} 17 \mathrm{C} \quad 109.5$

H17B-C17-H17C $\quad 109.5$

C15-C18-H18A $\quad 109.5$

$\mathrm{C} 15-\mathrm{C} 18-\mathrm{H} 18 \mathrm{~B} \quad 109.5$

$\mathrm{C} 15-\mathrm{C} 18-\mathrm{H} 18 \mathrm{C} \quad 109.5$

$\mathrm{H} 18 \mathrm{~A}-\mathrm{C} 18-\mathrm{H} 18 \mathrm{~B} \quad 109.5$

$\mathrm{H} 18 \mathrm{~A}-\mathrm{C} 18-\mathrm{H} 18 \mathrm{C} \quad 109.5$ 


$\begin{array}{ll}\mathrm{C} 12-\mathrm{C} 13-\mathrm{C} 17 & 122.0(2) \\ \mathrm{C} 11-\mathrm{C} 12-\mathrm{H} 12 & 118.6 \\ \mathrm{O} 1-\mathrm{C} 1-\mathrm{C} 6-\mathrm{C} 5 & -178.81(18) \\ \mathrm{O} 1-\mathrm{C} 1-\mathrm{C} 6-\mathrm{C} 9 & 1.4(3) \\ \mathrm{O} 1-\mathrm{C} 1-\mathrm{C} 2-\mathrm{C} 3 & -178.87(18) \\ \mathrm{O} 1-\mathrm{C} 1-\mathrm{C} 2-\mathrm{C} 7 & 2.7(3) \\ \mathrm{O} 2-\mathrm{C} 16-\mathrm{C} 15-\mathrm{C} 14 & 179.63(19) \\ \mathrm{O} 2-\mathrm{C} 16-\mathrm{C} 15-\mathrm{C} 18 & 0.6(3) \\ \mathrm{N} 1-\mathrm{C} 19-\mathrm{C} 24-\mathrm{C} 23 & 174.32(17) \\ \mathrm{N} 1-\mathrm{C} 19-\mathrm{C} 20-\mathrm{C} 21 & -171.58(15) \\ \mathrm{C} 11-\mathrm{C} 16-\mathrm{C} 15-\mathrm{C} 14 & 0.5(3) \\ \mathrm{C} 11-\mathrm{C} 16-\mathrm{C} 15-\mathrm{C} 18 & -178.5(2) \\ \mathrm{C} 1-\mathrm{C} 6-\mathrm{C} 5-\mathrm{C} 4 & -2.0(3) \\ \mathrm{C} 1-\mathrm{C} 6-\mathrm{C} 9-\mathrm{N} 1 & -43.1(2) \\ \mathrm{C} 4-\mathrm{C} 3-\mathrm{C} 2-\mathrm{C} 1 & -2.8(3) \\ \mathrm{C} 4-\mathrm{C} 3-\mathrm{C} 2-\mathrm{C} 7 & 175.6(2) \\ \mathrm{C} 16-\mathrm{C} 11-\mathrm{C} 10-\mathrm{N} 1 & 106.6(2) \\ \mathrm{C} 16-\mathrm{C} 11-\mathrm{C} 12-\mathrm{C} 13 & 1.5(3) \\ \mathrm{C} 6-\mathrm{C} 1-\mathrm{C} 2-\mathrm{C} 3 & 1.5(3) \\ \mathrm{C} 6-\mathrm{C} 1-\mathrm{C} 2-\mathrm{C} 7 & -176.93(19) \\ \mathrm{C} 5-\mathrm{C} 4-\mathrm{C} 3-\mathrm{C} 2 & 1.8(3) \\ \mathrm{C} 5-\mathrm{C} 6-\mathrm{C} 9-\mathrm{N} 1 & 137.12(19) \\ \mathrm{C} 9-\mathrm{N} 1-\mathrm{C} 19-\mathrm{C} 24 & -74.0(2) \\ \mathrm{C} 9-\mathrm{N} 1-\mathrm{C} 19-\mathrm{C} 20 & 158.55(16) \\ \mathrm{C} 9-\mathrm{N} 1-\mathrm{C} 10-\mathrm{C} 11 & -79.0(2) \\ \mathrm{C} 9-\mathrm{C} 6-\mathrm{C} 5-\mathrm{C} 4 & 177.78(19) \\ \mathrm{C} 19-\mathrm{N} 1-\mathrm{C} 9-\mathrm{C} 6 & -64.0(2) \\ \mathrm{C} 19-\mathrm{N} 1-\mathrm{C} 10-\mathrm{C} 11 & 152.53(16) \\ & \end{array}$

H18B-C18-H18C

$\mathrm{C} 19-\mathrm{C} 24-\mathrm{C} 23-\mathrm{C} 22$

$\mathrm{C} 19-\mathrm{C} 20-\mathrm{C} 21-\mathrm{C} 22$

C10-N1-C9-C6

$\mathrm{C} 10-\mathrm{N} 1-\mathrm{C} 19-\mathrm{C} 24$

$\mathrm{C} 10-\mathrm{N} 1-\mathrm{C} 19-\mathrm{C} 20$

$\mathrm{C} 10-\mathrm{C} 11-\mathrm{C} 16-\mathrm{O} 2$

$\mathrm{C} 10-\mathrm{C} 11-\mathrm{C} 16-\mathrm{C} 15$

$\mathrm{C} 10-\mathrm{C} 11-\mathrm{C} 12-\mathrm{C} 13$

$\mathrm{C} 3-\mathrm{C} 4-\mathrm{C} 5-\mathrm{C} 6$

$\mathrm{C} 24-\mathrm{C} 19-\mathrm{C} 20-\mathrm{C} 21$

$\mathrm{C} 14-\mathrm{C} 13-\mathrm{C} 12-\mathrm{C} 11$

$\mathrm{C} 13-\mathrm{C} 14-\mathrm{C} 15-\mathrm{C} 16$

C13-C14-C15-C18

$\mathrm{C} 12-\mathrm{C} 11-\mathrm{C} 16-\mathrm{O} 2$

$\mathrm{C} 12-\mathrm{C} 11-\mathrm{C} 16-\mathrm{C} 15$

$\mathrm{C} 12-\mathrm{C} 11-\mathrm{C} 10-\mathrm{N} 1$

$\mathrm{C} 2-\mathrm{C} 1-\mathrm{C} 6-\mathrm{C} 5$

$\mathrm{C} 2-\mathrm{C} 1-\mathrm{C} 6-\mathrm{C} 9$

$\mathrm{C} 20-\mathrm{C} 19-\mathrm{C} 24-\mathrm{C} 23$

$\mathrm{C} 15-\mathrm{C} 14-\mathrm{C} 13-\mathrm{C} 12$

$\mathrm{C} 15-\mathrm{C} 14-\mathrm{C} 13-\mathrm{C} 17$

$\mathrm{C} 21-\mathrm{C} 22-\mathrm{C} 23-\mathrm{C} 24$

$\mathrm{C} 23-\mathrm{C} 22-\mathrm{C} 21-\mathrm{C} 20$

$\mathrm{C} 8-\mathrm{C} 4-\mathrm{C} 5-\mathrm{C} 6$

$\mathrm{C} 8-\mathrm{C} 4-\mathrm{C} 3-\mathrm{C} 2$

$\mathrm{C} 17-\mathrm{C} 13-\mathrm{C} 12-\mathrm{C} 11$
109.5

$57.3(2)$

$-55.9(2)$

$166.26(16)$

$53.1(2)$

$-74.3(2)$

$-1.2(3)$

$177.75(18)$

$-177.36(18)$

0.7 (3)

$58.1(2)$

$-1.4(3)$

$-0.5(3)$

178.5 (2)

$179.99(19)$

$-1.0(3)$

$-74.6(2)$

$0.8(3)$

$-178.92(18)$

$-58.2(2)$

$0.9(3)$

-177.40 (19)

$-55.9(2)$

$54.7(2)$

$177.6(2)$

$-175.1(2)$

$176.86(19)$

Hydrogen-bond geometry $\left(\AA,{ }^{\circ}\right)$

\begin{tabular}{lllll}
\hline$D-\mathrm{H} \cdots A$ & $D-\mathrm{H}$ & $\mathrm{H} \cdots A$ & $D \cdots A$ & $D-\mathrm{H} \cdots A$ \\
\hline $\mathrm{O} 1-\mathrm{H} 1 \cdots \mathrm{N} 1$ & $0.89(4)$ & $1.81(4)$ & $2.630(2)$ & $153(3)$ \\
$\mathrm{O} 2-\mathrm{H} 2 \cdots \mathrm{O} 1^{\mathrm{i}}$ & $0.99(4)$ & $1.87(4)$ & $2.741(2)$ & $145(3)$ \\
$\mathrm{C} 7-\mathrm{H} 7 A \cdots \mathrm{O} 1$ & 0.98 & 2.40 & $2.854(3)$ & 108 \\
\hline
\end{tabular}

Symmetry code: (i) $x-1 / 2,-y+1 / 2, z$. 